\title{
A family of quadrics
}

\author{
By L. M. Brown.
}

(Received 22nd May, 1938. Read 4th June, 1938.)

In a recent paper ${ }^{1}$ I have discussed the families of quadrics in $[2 n]$ which are obtained by causing the members to have the greatest possible number of fixed [n-1]'s or "generators." It was found possible to fix four [ $[n-1]$ 's in general position; the family of quadrics through these possessed a "base" variety, common to all the members, which consisted of a highly degenerate $V_{n-1}$. Here I consider the same problem for quadrics in [2n+1], find how many generators may be assigned arbitrarily and discuss the common part of the quadrics which pass through such generators.

Now quadrics in a $[2 n+1] S$ have generating [n]'s. The freedom of the quadrics in $S$ is $(2 n+1)(n+2)$ and the postulation of an $[n]$ for quadrics is $\frac{1}{2}(n+1)(n+2)$, so that the greatest number of $[n]$ 's which can be independently assigned to a quadric in $S$ is the greatest integer in $(2 n+1)(n+2) / \frac{1}{2}(n+1)(n+2)$, i.e. in $4-2 /(n+1)$, and this is clearly always 3 for positive $n$. We shall therefore consider the family of quadrics in $S$ restricted to contain three generally placed [n]'s, and our problem will be to find the complete intersection, or "base," of the quadrics of the family. We observe that the problem is not directly analogous to that described in our previous paper; it is in fact much simpler, as the degeneracy which caused complication there no longer exists, the base consisting of a single variety.

The freedom of the family of quadrics is $\frac{1}{2}(n+2)(n-1)$ so that the base might be expected to be a variety of dimension $2 n-\frac{1}{2} n(n+1)$. But it is found in fact to consist of a variety of dimension $n+1$, that is, the dimension of the base is higher than might be expected from the freedom of the family. The base variety is in fact the dual of one of the "determinantal" manifolds studied by Room². Furthermore it is found that through any point of the

${ }^{1}$ L. M. Brown, Proc. Edinuurgh Math. Soc. (2), 5 (1938), 125.

2 See in particular T. G. Room, Proc. London Math. Soc. (2), 36 (1983), 1. In the notation of that paper the base variety is $)|n+1,2|,[2 n+1]($. 
variety there goes one line and also one $[n]$; varieties of this type have been widely discussed ${ }^{1}$.

One minor point may be settled immediately; let us refer for simplicity to the case of a quadric in [5]. If this is a genuine quadric (i.e. not a cone), it contains two systems of generating planes, and any two planes in the same system will intersect. It follows that it is impossible to find three generators of a genuine quadric in [5] which are all skew, for at least two must belong to the same system; and consequently through three planes in general position in [5] there are no genuine quadrics (they are in fact all cones with line vertices, the lines being the doubly-infinite set meeting the three planes). Nevertheless, we shall continue to consider three planes in general position, and not concern ourselves with the genuineness or otherwise of the quadrics through them, and similarly in other cases where the same point may arise.

1. Let $p, q, r$ be three $[n]$ 's in a $[2 n+1] S$. Through $r$ take any $[n+1] \Gamma$. It cuts $p$ and $q$ in points $P$ and $Q$, and in no position can it cut in more, for if, say $\Gamma$ cut $p$ in a line $l$, then $l$ must cut $r$, and $p$ and $r$ would not be skew. The line $P Q$ cuts $r$ in a point $R$. Now any quadric through $\Gamma$ must be a line-cone with vertex $P Q$ (or perhaps a cone of higher type whose vertex contains $P Q$ ), so let us project from $P Q$ into a $[2 n-1] \Sigma$. Then $p, q, \Gamma$ project into three $[n-1]$ 's $p^{\prime}, q^{\prime}$, $r^{\prime}$ in $\Sigma$, and these must be skew for all positions of $\Gamma$. Now for a general position of $\Gamma$ the number of linearly independent quadrics through such a triad is $\frac{1}{2} n(n-1)$, so the number of linearly independent quadrics through $\Gamma$ is also $\frac{1}{2} n(n-1)$. But since the number of linearly independent quadrics in the whole family is $\frac{1}{2} n(n+1)$ it follows that there are $n$ linearly independent quadrics not through $\Gamma$. These cut $\Gamma$ in quadrics of $\Gamma$ through $r$ and through $P Q$; each therefore consists of $r$ and an [n] through $P Q$. But $n$ linearly independent $[n]$ 's in an $[n+1]$ cut in a line, so the quadrics cut in $r$ and in $P Q$; i.e. $\Gamma$ cuts the base in $r$ and $P Q$. Now $\Gamma$ was any $[n+1]$ through $r$, and so the base consists of such lines as $P Q$, i.e. of the locus of all the lines meeting $p, q, r$.

We have however to guard against the possibility that for some special position of $\Gamma$ the number of quadrics through it should be

${ }^{3}$ See H. W. Richmond and F. Bath, Proc. Cambridge Phil. Soc., 22 (1924), 319, where a bibliography is given; in our connection see in particular two papers by C. Segre, Rend. di Palermo, 5 (1891), 192 and 30 (1910), 87. 
more than the normal number $\frac{1}{2} n(n-1)$. We shall do this if we prove that $\frac{1}{2} n(n-1)$ is always the number of quadrics in $\Sigma$ through $p^{\prime}, q^{\prime}, r^{\prime}$ for any position of $p^{\prime}, q^{\prime}, r^{\prime}$ in which they remain skew.

The number of linearly independent quadrics in $\Sigma$ is $n(2 n+1)$. The postulation of a single $[n-1] p^{\prime}$ is $\frac{1}{2} n(n+1)$. If the postulation of a second $[n-1] q^{\prime}$ is less than $\frac{1}{2} n(n+1)$ it implies that any quadric $J$ through $p^{\prime}$ which is made to pass through any quadric $j$ of $q^{\prime}$ will contain $q^{\prime}$. Take $j$ to be two $[n-2]^{\prime}$ 's of $q^{\prime}, a$ and $b$. Then we may take as the quadric $J$ through $p^{\prime}, a, b$ the pair of [2n-2]'s consisting of $p^{\prime} a$ and any [2n- 2] through $b$. But unless $q^{\prime}$ meets $p^{\prime}$ this quadric $J$ does not contain $q^{\prime}$. Consequently if $p^{\prime}$ and $q^{\prime}$ are skew their combined postulation is $n(n+1)$. If now the additional postulation of a third $[n-1] r^{\prime}$ is less than $\frac{1}{2} n(n+1)$ it implies that if a quadric $K$ through $p^{\prime}$ and $q^{\prime}$ passes through a quadric $k$ of $r^{\prime}$, then $K$ must contain $r^{\prime}$. But if we take $k$ to be two [n-2]'s $c, d$ of $r^{\prime}$, we may take $K$ as the [' $2 n-2]$-pair $p^{\prime} c, q^{\prime} d$; and unless $r^{\prime}$ meets $p^{\prime}$ or $q^{\prime}$ this quadric $K$ does not contain $r^{\prime}$. Consequently the combined postulation of $p^{\prime}, q^{\prime}, r^{\prime}$ is $\frac{3}{2} n(n+1)$ and so by the customary argument the number of quadrics in $\Sigma$ through $p^{\prime}, q^{\prime}, r^{\prime}$ is $\frac{1}{2} n(n-1)$.

Now we have in the paper mentioned above shown that the locus of lines meeting three $[n]$ 's in $S$ is a $V_{n+1}$ of order $n+1$. Let us call it $v$.

The base consists of the single $V_{n+1} V$, of order $n+1$, the locus of the lines meeting $p, q, r$.

2. A most noteworthy property of the variety $V$ is that it is ruled not merely by lines, but also by $[n]$ 's. The lines of $V$ set up a projective relationship between the $[n]$ 's $p$ and $q$, and if $A_{1}, \ldots$, $A_{n+1}$ is any simplex in $p$ and $B_{1}, \ldots, B_{n+1}$ the corresponding simplex in $q$, then any point of $V$ may be written as

$$
\lambda\left(t_{1} A_{1}+\ldots+t_{n+1} A_{n+1}\right)+\mu\left(t_{1} B_{1}+\ldots+t_{n+1} B_{n+1}\right),
$$

when lines are obtained by varying $\lambda$ and $\mu$, and [n]'s are obtained by varying the parameters $t_{1} \ldots t_{n+1}$. In fact $V$ may be defined as the locus of lines joining corresponding points of two projectively related $[n]$ 's, or as the locus of $[n]$ 's joining corresponding points of $n+1$ projectively related lines. Room, in the reference cited and elsewhere, considers a large class of varieties obtained in this manner. In particular he finds a rational map of the dual of $V$. It is however easy to find a map of $V$ by direct projection. If $\widetilde{\sigma}$ is a general $[n-1]$ in $r$, and we project $\mathcal{V}$ from $\sigma$ into a skew $[n+1] T$, then any point 
of $V$ projects into a single point of $T$, and a general point of $T$ is projected from just one point of $V$; there is thus a map $(i . e$. a $(1,1)$ relationship) of $V$ on $T$. A general line of $T$ joined to $\sigma$ gives a general $[n+1] \lambda$ through $\sigma$, and this cuts $V$ in a conic. Now the prime-sections of $V$ cut this conic in two points, and therefore the maps in $T$ of the prime-sections are quadric primals of $T$; call them $\Phi$-quadrics. The $[n] r$ projects from $\tau$ into a point $P$ of $T$, and those lines of $V$ which meet $\varpi$ project into the points of an $[n-1] \rho$ of $T$. If $\lambda$ is an $[n+1]$ through $r$, then it cuts $V$ in one line outside of $r$, so the $\Phi$-quadrics all pass through $P$; and if $\lambda$ is an $[n+1]$ through $\pi$ and through a line $l$ of $V$ meeting $\sigma$, then $\lambda$ meets $V$ in $l$ and a second line $m$, so a line of $T$ meeting $\rho$ must represent a line, so all the $\Phi$-quadrics contain $\rho$. A simple freedom argument shows that the two conditions of passage through $P$ and $\rho$ are the sole conditions on the $\Phi$-quadrics of $T$. We may observe that if $n$ is more than 2 , the $\Phi$-quadrics will all be cones.

It is perhaps worth mention in conclusion that methods analogous to those employed above yield rational maps of certain of the other varieties considered in our previous paper. For example, the locus $V_{2}$ of planes in [9] meeting a [5] in a line and three [3]'s in points is a $V_{5}^{5}$; then a map of $V_{2}$ may be made in a [5] $T$ where the $\Phi$-primals are cubic primals passing through a $V_{3}^{3} U$ and having a line $l$ as doubleline, where $U$ is the locus of lines meeting three planes, and $l$ is a line of $U$.

The Royal Technical College, Glasgow, C. 1. 